\title{
Research on Evolutionary Model and Dynamic Analysis for Internet Public Opinion Spreading
}

\author{
Wei Zhang ${ }^{1}{ }^{1}$ and Yong Chen ${ }^{2}$ \\ ${ }^{1}$ School of Traffic and Transportation, Lanzhou Jiaotong University, Lanzhou 730070, China \\ ${ }^{2}$ School of Electronic and Information Engineering, Lanzhou Jiaotong University, Lanzhou 730070, China \\ Correspondence should be addressed to Wei Zhang; zhangw992@126.com
}

Received 20 July 2021; Revised 16 September 2021; Accepted 30 September 2021; Published 19 October 2021

Academic Editor: Giacomo Fiumara

Copyright $\odot 2021$ Wei Zhang and Yong Chen. This is an open access article distributed under the Creative Commons Attribution License, which permits unrestricted use, distribution, and reproduction in any medium, provided the original work is properly cited.

\begin{abstract}
Internet public opinion has developed rapidly in recent years, which has more influence on society. The relevant problems of Internet public opinion have become the hotspots of research. The complexity of Internet public opinion spreading brings difficulties to research and analysis. For the problems of Internet public opinion spreading, an evolutionary model of Internet public opinion spreading is built based on evolutionary game theory in this paper, considering three subjects including Internet media, Internet users, and government. Then, the evolutionary stable strategies of the Internet public opinion spreading system are studied. Finally, the influences of the stable strategy of the Internet public opinion spreading system are analyzed through simulation. Relevant conclusions are obtained. The results show that there are multiple possible stable strategies in the evolution of Internet public opinion. The different initial probabilities of the strategy lead to the different stable strategies of the evolutionary system. The stable strategy is influenced by some factors, such as the punished loss of Internet media, the gain of Internet users from government controlling, and the cost of government. These conclusions have a certain guiding significance to Internet public opinion management and control.
\end{abstract}

\section{Introduction}

Internet public opinion is a group of people's perceptions, attitudes, emotions, and behavioral tendencies of the event, which is transmitted through the Internet. Internet public opinion has grown up to be a major component of social public opinion and has an increasing impact on social stability. Internet public opinion has the characteristics of randomness, diversity, and deviation due to the openness and virtuality of the Internet, which complicates the study of Internet public opinion.

Many scholars have carried out research work on Internet public opinion, mainly focusing on the following four aspects. (1) The first aspect is qualitative analysis of Internet public opinion, mainly analyzing the causes of the generation and dissemination of public opinion, the impact on society, the challenges, and coping strategies. Lin analyzed the Internet public opinion of emergencies in recent years in
China from the five research aspects of communication, monitoring, control, response, and governance. He put forward the orientation and research approach of Internet public opinion governance [1]. Wang studied the communication path of enterprise crisis network public opinion and its vertical spillover effect in the supply chain [2]. Mei carried out evaluating the risk levels of Internet public opinion and made a classification of risk levels [3]. Stockmann explained how major social media differ in terms of their structure and the company's motivation and specified which features are more likely to facilitate the emergence of online public opinion in Chinese social media [4]. (2) The second aspect is empirical analysis of Internet public opinion. For a specific case, the dissemination subject and information dissemination characteristics are analyzed, and the development characteristics are obtained to give prevention and response plans. Silje analyzed the evolution of public opinion about nuclear energy in Switzerland after the accident at 
Fukushima Daiichi Nuclear Power Plant [5]. Tian analyzed deeply the prism refraction generating logic and the content level of emotion attitude opinion and behavior orientation according to grounded theory, taking the taxi strike incident in 2015 as a research case [6]. Li studied evolution mechanism, path, and motivation of Internet public opinion, taking animal epidemic crisis as an example [7]. Nip examined the prominence of various user categories as opinion leaders in 29 corruption cases exposed on Sina Weibo and showed that news organizations and online media were the main opinion leaders [8]. (3) The third aspect is studying related technologies in the process of public opinion monitoring, such as data acquisition, data processing, data extraction, and text classification technology [9-11]. Wang built a wide stationary time series model of network public opinion and proposed a network public opinion supervision forecast algorithm based on large data semantic feature analysis [12]. Ma studied a fast classification algorithm of parallel naive Bayesian for network public opinion based on Hadoop platform [13]. Guo built a model to predict the evolution trend of network public opinion by using the theory and method of extension clustering [14]. Sandra applied different classifiers to extract information about the affective tone of public opinion in online communication [15]. (4) The fourth aspect is studying the spread of Internet public opinion. The characteristics and development trend of public opinion are analyzed by establishing various models [16-18]. The regulations and influencing factors of public opinion development are obtained, which provide guidance for public opinion control. Javier established network models of minority opinion spreading based on the agent method and analyzed the phenomena of minority opinion spreading [19]. Zhao built the model of microblog public opinion dissemination trend and analyzed the influencing factors of microblog public opinion dissemination based on information ecology theory [20]. Zhou proposed the concepts of public opinion in self-media network, built a public opinion diffusion model based on forwarding effect, and obtained the characteristics of the public opinion spreading by simulation [21]. These research studies explained the occurrence and mechanism of Internet public opinion $[22,23]$ and verified that some methods can be used to monitor, forecast, and control it better [24-28]. The research studies on the spread of Internet public opinion have an important significance. The relative rules and mechanisms can help us to manage Internet public opinion better. The above research studies on Internet public opinion have achieved corresponding results. However, the works are mainly about qualitative research and empirical research, and technology research studies are relatively less. The study on the spread of Internet public opinion is focussed on the spread characteristics, trends, influencing factors, and so on.

Evolutionary game theory is one of the important fields of modern game theory [29]. Evolutionary game theory is on the premise of the bound rationality, which is closer to human. It overcomes the situation that completely rational game analysis separates from practice. It has been applied in various fields and has a very important role and value [30-33].
For the problem of the spread of Internet public opinion, this paper establishes an evolutionary model of Internet public opinion spreading based on evolutionary game theory. The model considers three subjects including Internet media, Internet users, and government. The evolutionary stable strategies are analyzed, and the influences of the stable strategies are studied. The evolutionary characteristics and regulations are obtained at last. These conclusions can provide guidance for Internet public opinion prediction and control.

\section{Modelling and Analysis}

2.1. Problem Description. When an event occurs, Internet media will publish relevant information. Internet users will concern about them. Reporters, reviewers, and columnists write many essays on the Internet. These essays have guidance roles. However, sometimes it may induce the public's bad mood, cause the people's illegal and radical behavior, and then pose a threat to social stability. At this time, the government must intervene and control, such as investigating the facts and restoring the truth, which meets the psychology of Internet users, and finally Internet public opinion is subsided. The problem of Internet public opinion evolution involves three subjects: Internet media, Internet user, and government. In the evolution system of Internet public opinion, Internet media plays a role of publishing information, Internet user concerns information, and government is a role of control. Internet media has two strategies to choose: publishing information and no-publishing information. Internet user has two strategies: concerning information and no-concerning information. Government has two strategies: controlling public opinion and no-controlling public opinion. Next, a model is established based on evolution game theory, to analyze the evolution of Internet public opinion, the influence factors of evolution, and stable strategies of three subjects.

2.2. Evolution Game Model of the Internet Public Opinion Spreading System. The relevant variables of the model are defined as follows:

$R^{M}$ means the gain of Internet media when Internet media publishes information on the Internet and Internet users concern the information.

$C^{M}$ means the cost of publishing information for Internet media.

$W^{M}$ means the loss of Internet media when Internet media publishes information and the government controls it. If the information Internet media published distorted facts, the information guidance does not agree with that of government; the Internet media will be punished by government.

$R_{1}^{H}$ means the information gain of concerning information for Internet users.

$R_{2}^{H}$ means the gain of social stability obtained by Internet users when the government controls an Internet public opinion. Government actively resolves public 
opinion and maintains social stability. Internet users will benefit from it.

$C^{H}$ means the cost of collecting and concerning information on the Internet for Internet users.

$R^{G}$ means the positive impact of government social image when the government takes measures to control Internet public opinion.

$C^{G}$ means the cost of government controlling Internet public opinion.

$W^{G}$ means the loss of government when the government does not take measures of controlling Internet public opinion. If the government does not control the Internet public opinion and lets it grow, it will bring a threat to social stability and cause a negative impact on society.

$M_{1}$ means the strategy of publishing information for Internet media.

$M_{2}$ means the strategy of no-publishing information for Internet media.

$H_{1}$ means the strategy of concerning information for Internet user.

$\mathrm{H}_{2}$ means the strategy of no-concerning information for Internet user.

$G_{1}$ means the strategy of controlling Internet public opinion for government.

$G_{2}$ means the strategy of no-controlling Internet public opinion for government.

Internet media, Internet user, and government are three subjects of the evolution system. Every subject faces with two strategies to choose. Internet media may choose publishing information or no-publishing information. Internet user may choose concerning information or no-concerning information. Government may choose controlling Internet public opinion or no-controlling Internet public opinion. The choice of strategy not only is related to the individual but also is impacted by other subjects. It is a game process. The game model of the three subjects is established. The specific gain matrix is shown in Table 1. Table 1 lists gains of the three subjects under the condition of different strategy combinations.

2.3. Strategy Analysis of Internet Media. Suppose the probability of Internet media choosing the publishing strategy is $x$, the probability of Internet user choosing the concerning strategy is $y$, and the probability of the government choosing the controlling strategy is $z$.

For Internet media, the expected gain of publishing strategy is

$$
\begin{aligned}
U_{M 1}= & \left(R^{M}-C^{M}-W^{M}\right) y z+\left(R^{M}-C^{M}\right) y(1-z) \\
& +\left(-C^{M}-W^{M}\right)(1-y) z+\left(-C^{M}\right)(1-y)(1-z) \\
= & R^{M} y-W^{M} z-C^{M} .
\end{aligned}
$$

TABLE 1: Gain matrix of the three subjects.

\begin{tabular}{lccc}
\hline Strategy & Internet media & Internet user & Government \\
\hline$\left(M_{1}, H_{1}, G_{1}\right)$ & $R^{M}-C^{M}-W^{M}$ & $R_{1}^{H}-C^{H}+R_{2}^{H}$ & $R^{G}-C^{G}+W^{M}$ \\
$\left(M_{1}, H_{1}, G_{2}\right)$ & $R^{M}-C^{M}$ & $R_{1}^{H}-C^{H}$ & $-W^{G}$ \\
$\left(M_{1}, H_{2}, G_{1}\right)$ & $-C^{M}-W^{M}$ & $R_{2}^{H}$ & $R^{G}-C^{G}+W^{M}$ \\
$\left(M_{1}, H_{2}, G_{2}\right)$ & $-C^{M}$ & 0 & $-W^{G}$ \\
$\left(M_{2}, H_{1}, G_{1}\right)$ & $-R^{M}$ & $-C^{H}+R_{2}^{H}$ & $R^{G}-C^{G}$ \\
$\left(M_{2}, H_{1}, G_{2}\right)$ & $-R^{M}$ & $-C^{H}$ & $-W^{G}$ \\
$\left(M_{2}, H_{2}, G_{1}\right)$ & 0 & 0 & $-C^{G}$ \\
$\left(M_{2}, H_{2}, G_{2}\right)$ & 0 & 0 & 0 \\
\hline
\end{tabular}

Expected gain of no-publishing strategy is

$$
U_{M 2}=\left(-R^{M}\right) y z+\left(-R^{M}\right) y(1-r)=-R^{M} y .
$$

Average expected gain is

$$
\begin{aligned}
\bar{U}_{M} & =U_{M 1} x+U_{M 2}(1-x) \\
& =2 R^{M} y x-R^{M} y-W^{M} z x-C^{M} x .
\end{aligned}
$$

The replicator dynamic equation of Internet medias is

$$
\begin{aligned}
F(x) & =\frac{\mathrm{d} x}{\mathrm{~d} t}=x\left(U_{M 1}-\bar{U}_{M}\right) \\
& =x(1-x)\left(2 R^{M} y-C^{M}-W^{M} z\right) .
\end{aligned}
$$

Suppose $F(x)=(\mathrm{d} x / \mathrm{d} t)=0$, then $x=0, x=1$, and $z^{*}=\left(2 R^{M} y-C^{M}\right) / W^{M}$.

The stability analysis is as follows:

(1) If $z=z^{*}$, then $F(x)=0$ and $F^{\prime}(x)=0$. Here, any strategy may be stable.

(2) If $z<z^{*}$, then $F^{\prime}(0)>0$ and $F^{\prime}(1)<0$. Here, $x=1$ is stable strategy. At this time, Internet media will choose the publishing strategy.

(3) If $z>z^{*}$, then $F^{\prime}(0)<0$ and $F^{\prime}(1)>0$. Here, $x=0$ is stable strategy. At this time, Internet media will choose no-publishing strategy.

Based on the above analysis, the strategy of Internet media depends on the initial probability of the strategy of Internet users and government. Strategy dynamic evolution and stability of Internet media are shown in Figure 1. The shading $z_{0}=z^{*}$ of Figure 1 divides the space into upper and lower parts. When the initial probability is above the shading, the stable strategy of Internet media is $x=0$; that is, Internet media will choose no-publishing strategy. When the initial probability is below the shading, the stable strategy of Internet media is $x=1$; that is, Internet media will choose the publishing strategy.

2.4. Strategy Analysis of Internet User. For Internet user, the expected gain of concerning strategy is

$$
\begin{aligned}
U_{H 1}= & \left(R_{1}^{H}-C^{H}+R_{2}^{H}\right) x z+\left(R_{1}^{H}-C^{H}\right) x(1-z) \\
& +\left(R_{2}^{H}-C^{H}\right)(1-x) z-C^{H}(1-x)(1-z) \\
= & R_{2}^{H} z+R_{1}^{H} x-C^{H} .
\end{aligned}
$$




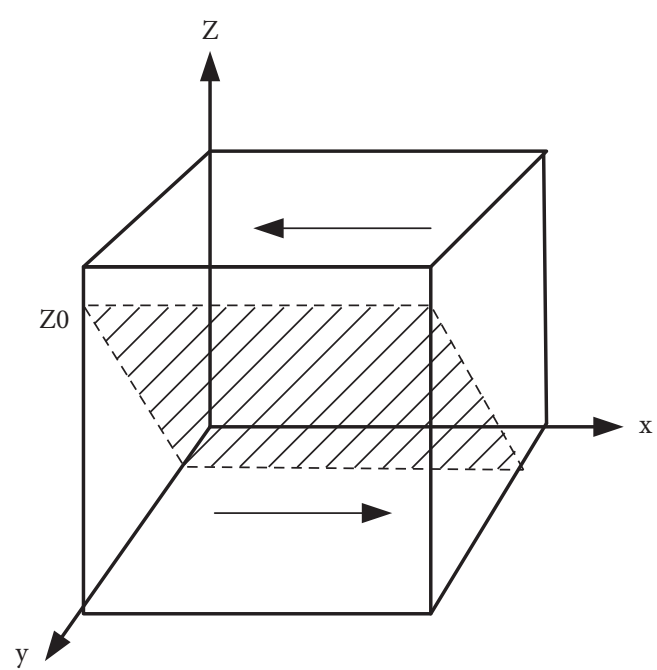

FIgURE 1: Strategy dynamic evolution and stability of Internet media.

Expected gain of no-concerning strategy is

$$
U_{H 2}=R_{2}^{H} x z \text {. }
$$

Average expected gain is

$$
\begin{aligned}
\bar{U}_{H} & =U_{H 1} y+U_{H 2}(1-y) \\
& =R_{2}^{H} y z+R_{1}^{H} x y-C^{H} y+R_{2}^{H} x z-R_{2}^{H} x y z .
\end{aligned}
$$

The replicator dynamic equation of Internet users is

$$
\begin{aligned}
F(y) & =\frac{\mathrm{d} y}{\mathrm{~d} t}=y\left(U_{H 1}-\bar{U}_{H}\right) \\
& =y(1-y)\left(R_{1}^{H} x-R_{2}^{H} x z+R_{2}^{H} z-C^{H}\right) .
\end{aligned}
$$

Suppose $F(y)=(\mathrm{d} y / \mathrm{d} t)=0$, then $y=0, y=1$, and $x^{*}=\left(C^{H}-R_{2}^{H} z\right) /\left(R_{1}^{H}-R_{2}^{H} z\right)$.

The stability analysis is as follows:

(1) When $C^{H}>R_{1}^{H}$

(1) If $x=x^{*}$, then $F(y)=0$ and $F^{\prime}(y)=0$. Here, any strategy may be stable.

(2) If $x>x^{*}$, then $F^{\prime}(0)>0$ and $F^{\prime}(1)<0$. Here, $y=1$ is stable strategy. At this time, the Internet user will choose the concerning strategy.

(3) If $x<x^{*}$, then $F^{\prime}(0)<0$ and $F^{\prime}(1)>0$. Here, $y=0$ is stable strategy. At this time, the Internet user will choose no-concerning strategy.

(2) When $C^{H}<R_{1}^{H}$

(1) If $x=x^{*}$, then $F(y)=0$ and $F^{\prime}(y)=0$. Here, any strategy may be stable.

(2) If $x>x^{*}$, then $F^{\prime}(0)>0$ and $F^{\prime}(1)<0$. Here, $y=1$ is stable strategy. At this time, the Internet user will choose the concerning strategy.

(3) If $x<x^{*}$, then $F^{\prime}(0)<0$ and $F^{\prime}(1)>0$. Here, $y=0$ is stable strategy. At this time, the Internet user will choose no-concerning strategy.
(3) When $C^{H}=R_{1}^{H}$

(1) If $x=1$, then $F(y)=0$ and $F^{\prime}(y)=0$. Here, any strategy may be stable.

(2) If $x \neq 1$, then $F^{\prime}(0)>0$ and $F^{\prime}(1)<0$. Here, $y=$ 0 is stable strategy. Internet user will choose noconcerning strategy.

Strategy dynamic evolution and stability of Internet users are shown in Figure 2. Figure 2(a) shows the situation under the condition of $C^{H}>R_{1}^{H}$. Figure 2(b) shows the situation under the condition of $C^{H}<R_{1}^{H}$. Figure 2(c) shows the situation under the condition of $C^{H}=R_{1}^{H}$. The strategy of Internet users depends on the initial probability of the strategy of Internet media and government. The shading $x_{0}=x^{*}$ of Figure 2 divides the space into upper and lower parts. When the initial probability is above the shading, the stable strategy of Internet users is $y=1$; that is, Internet users will choose the concerning strategy. When the initial probability is below the shading, the stable strategy of Internet users is $y=0$; that is, Internet users will choose noconcerning strategy.

2.5. Strategy Analysis of Government. For the government, the expected gain of controlling strategy is

$$
\begin{aligned}
U_{G 1}= & \left(R^{G}-C^{G}+W^{M}\right) x y+\left(R^{G}-C^{G}+W^{M}\right) x(1-y) \\
& +\left(R^{G}-C^{G}\right)(1-x) y-C^{G}(1-x)(1-y) \\
= & R^{G} x+W^{M} x+R^{G} y-R^{G} x y-C^{G} .
\end{aligned}
$$

Expected gain of no-controlling strategy is

$$
\begin{aligned}
U_{G 2} & =-W^{G} x y-W^{G} x(1-y)-W^{G}(1-x) y \\
& =-W^{G}(x+y-x y) .
\end{aligned}
$$

Average expected gain is

$$
\begin{aligned}
\bar{U}_{G}= & U_{G 1} z+U_{G 2}(1-z) \\
= & \left(R^{G} x+W^{M} x+R^{G} y-R^{G} x y-C^{G}\right) z \\
& -W^{G}(x+y-x y)(1-z) .
\end{aligned}
$$

The replicator dynamic equation of government is

$$
\begin{aligned}
F(z)= & \frac{\mathrm{d} z}{\mathrm{~d} t}=z\left(U_{G 1}-\bar{U}_{G}\right) \\
= & z(1-z)\left[\left(R^{G}+W^{G}+W^{M}\right) x+\left(R^{G}+W^{G}\right) y\right. \\
& \left.-\left(R^{G}+W^{G}\right) x y-C^{G}\right] .
\end{aligned}
$$

Suppose $F(z)=(\mathrm{d} z / \mathrm{d} t)=0$, then $z=0, z=1$, and $y^{*}=\left(C^{G}-\left(R^{G}+W^{M}+W^{G}\right) x\right) /\left(R^{G}+W^{G}\right)(1-x)$.

The stability analysis is as follows:

(1) When $C^{G}>R^{G}+W^{G}+W^{M}$ 


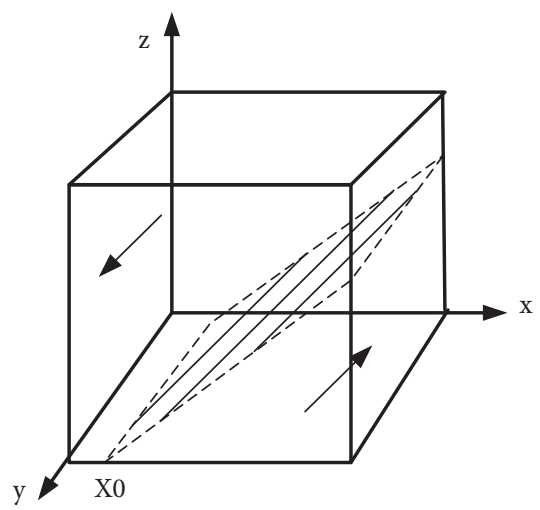

(a)

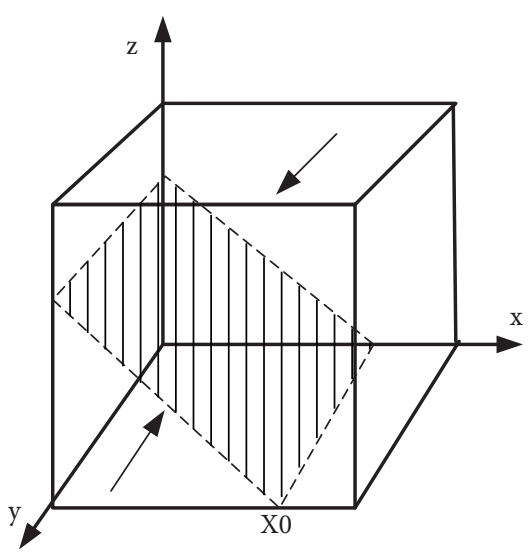

(b)

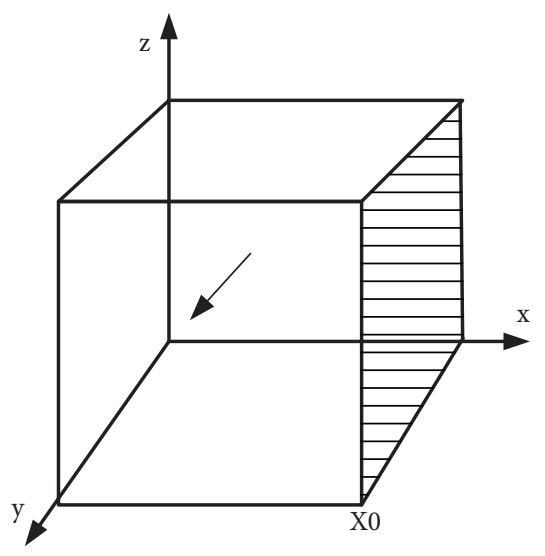

(c)

FIgURE 2: Strategy dynamic evolution and stability of Internet users: (a) $C^{H}>R_{1}^{H}$; (b) $C^{H}<R_{1}^{H}$; (c) $C^{H}=R_{1}^{H}$.

(1) If $y=y^{*}$, then $F(z)=0$ and $F^{\prime}(z)=0$. Here, any strategy may be stable.

(2) If $y>y^{*}$, then $F^{\prime}(0)>0$ and $F^{\prime}(1)<0$. Here, $z=$ 1 is stable strategy. At this time, the government will choose the controlling strategy.

(3) If $y<y^{*}$, then $F^{\prime}(0)<0$ and $F^{\prime}(1)>0$. Here, $z=$ 0 is stable strategy. At this time, the government will choose no-controlling strategy.

(2) When $C^{G}<R^{G}+W^{G}+W^{M}$

(1) If $y=y^{*}$, then $F(z)=0$ and $F^{\prime}(z)=0$. Here, any strategy may be stable.

(2) If $y>y^{*}$, then $F^{\prime}(0)>0$ and $F^{\prime}(1)<0$. Here, $z=$ 1 is stable strategy. At this time, the government will choose the controlling strategy.

(3) If $y<y^{*}$, then $F^{\prime}(0)<0$ and $F^{\prime}(1)>0$. Here, $z=$ 0 is stable strategy. At this time, the government will choose no-controlling strategy.

(3) When $C^{G}=R^{G}+W^{G}+W^{M}$

(1) If $W^{M}=0$, then $y^{*}=1$.

(a) If $y=1$, then $F(z)=0$ and $F^{\prime}(z)=0$. Here, any strategy may be stable.

(b) If $y \neq 1$, then $F^{\prime}(0)>0$ and $F^{\prime}(1)<0$. Here, $z=0$ is stable strategy. Government will choose nocontrolling strategy.

(2) If $W^{M} \neq 0$, then $y^{*}>1$ and $y<y^{*}$. Here, $z=0$ is stable strategy. Government will choose nocontrolling strategy.

Strategy dynamic evolution and stability of government are shown in Figure 3. Figure 3(a) shows the situation under the condition of $C^{G}>R^{G}+W^{G}+W^{M}$. Figure $3(\mathrm{~b})$ shows the situation under the condition of $C^{G}<R^{G}+W^{G}+W^{M}$. Figure 3(c) shows the situation under the condition of $C^{G}=R^{G}+W^{G}+W^{M}$. The strategy of government depends on the initial probability of the strategy of Internet media and Internet users. The shading $y_{0}=y^{*}$ of Figure 3 divides the space into upper and lower parts. When the initial probability is above the shading, the stable strategy of the government is $z=1$; that is, the government will choose the controlling strategy. When the initial probability is below the shading, the stable strategy of the government is $z=0$; that is, the government will choose no-controlling strategy.

2.6. Stable Strategy Analysis of Evolution System. Based on the above analysis, suppose the space $z>z^{*}$ is space I, the space $z<z^{*}$ is space II, the space $x>x^{*}$ is space III, the space $x<x^{*}$ is space IV, the space $y>y^{*}$ is space $\mathrm{V}$, and the space $y<y^{*}$ is space VI. Comprehensively considering Internet media, Internet users, and government, $x^{*}, y^{*}$, and $z^{*}$ divide the whole space into eight parts. The stable strategies of every subspace are shown in Table 2. For example, if the initial probability of the strategy of Internet media falls in the space III, that of Internet user falls in the space $\mathrm{V}$ and that of government falls in the space I, the stable strategy is $(1,1,1)$. That is, the stable strategy of Internet media is the publishing strategy, the stable strategy of Internet user is the concerning strategy, and the stable strategy of government is controlling strategy.

\section{Numerical Simulation Analysis}

Suppose the cost of publishing the relative information for Internet media after an event happens is $C^{M}=100$. The information gain of concerning information for Internet user is $R_{1}^{H}=60$. The gain of Internet media is $R^{M}=200$ when Internet users concern the information on the Internet published by Internet media. The cost of collecting and concerning information on the Internet for Internet user is $C^{H}=50$. If the government takes measures of controlling Internet public opinion, the cost is $C^{G}=200$. The gain of government social image is $R^{G}=200$. The gain of social stability obtained by Internet users is $R_{2}^{H}=100$. When the information published by Internet media does not agree with that of government, the Internet media will be punished and the loss of Internet media is $W^{M}=200$. If government does not take measures of controlling Internet public opinion, the loss of government is $W^{G}=300$ due to social instability of social caused by Internet public opinion. Under 


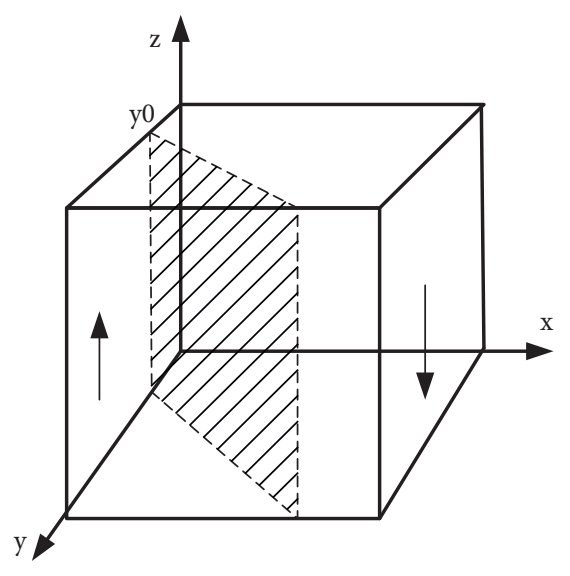

(a)

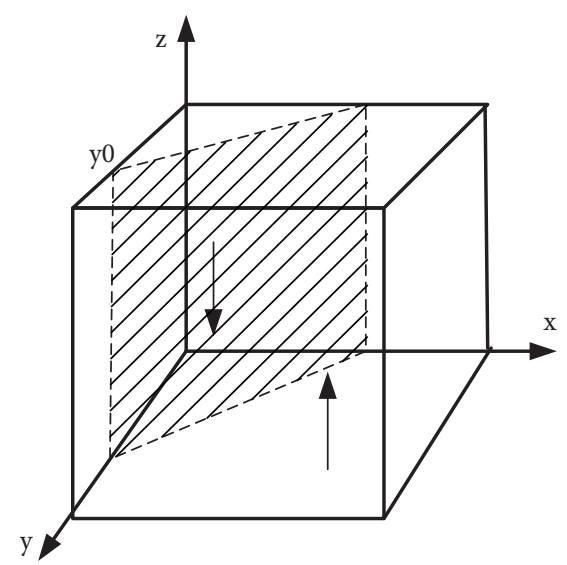

(b)

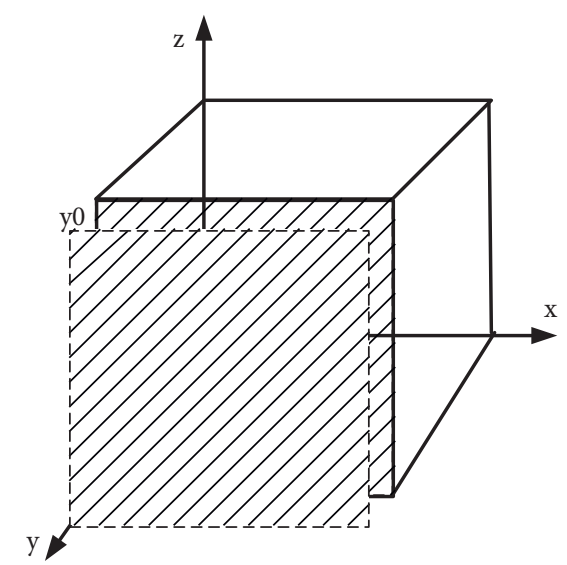

(c)

FIGURE 3: Strategy dynamic evolution and stability of government: (a) $C^{G}>R^{G}+W^{G}+W^{M}$; (b) $C^{G}<R^{G}+W^{G}+W^{M}$; (c) $C^{G}=R^{G}+W^{G}+W^{M}$.

TABLE 2: The stable strategy of subspaces.

\begin{tabular}{lccc}
\hline Subspace & Stable strategy & Subspace & Stable strategy \\
\hline (I, III, V) & $(1,1,1)$ & (II, III, V) & $(0,1,1)$ \\
(I, III, VI) & $(1,1,0)$ & (II, III, VI) & $(0,1,0)$ \\
(I, IV, V) & $(1,0,1)$ & (II, IV, V) & $(0,0,1)$ \\
(I, IV, VI) & $(1,0,0)$ & (II, IV, VI) & $(0,0,0)$ \\
\hline
\end{tabular}

the above conditions, the strategy dynamic evolution and influence of stable strategy are analyzed by simulation.

\subsection{The Strategy Dynamic Evolution of Different Initial} Probabilities. The stable strategy is different with different initial probabilities of the strategies. When the initial probability of the strategies of three subjects is $(0.5,0.5,0.5)$, the stable strategy is $(1,1,1)$, as shown in Figure $4(a)$. Government becomes stable quickly, closely followed by Internet user. The probability of Internet media choosing publishing strategy decreases first then increases. At first, the government chooses the controlling strategy, so the probability of Internet media choosing publishing strategy decreases. However, with the increase in probability of Internet user choosing concerning information, the probability of Internet media choosing publishing strategy increases sharply to 1 . At this time, the system reaches stability. Internet media will select the publishing information strategy, Internet users will select the concerning information strategy, and the government will select the controlling strategy. The initial probability of the strategy is changed without changing other conditions. When the initial probability of the strategy is $(0.2,0.2,0.5)$, the strategies dynamic evolution is shown in Figure 4(b). The initial probability of Internet media choosing the publishing strategy and that of Internet users choosing the concerning strategy are both decreased to 0.2 . At this time, the stable strategy is $(0,0,0)$. Internet media will not select the publishing information strategy, Internet users will not select the concerning information strategy, and the government will not select the controlling strategies.
3.2. The Influence Analysis of $W^{M}$ on Strategy. The punishment $W^{M}$ has an important impact on the stability strategy. Next, the effect of the value on the stability strategy is analyzed by simulation. The value of $W^{M}$ is changed without changing other conditions to analyze the influence. When the value of $W^{M}$ is lower than 250 , the strategy dynamic evolution is shown in Figure 5(a). The stable strategy is (1, 1, 1). Internet users and government reach stability quickly, and Internet users reach stability relatively slowly. When $W^{M}$ is increased to 250 , the strategy dynamic evolution is shown in Figure 5(b). Although the stable strategy is $(1,1,1)$, Internet users reach stability more quickly and the probability of Internet media choosing the publishing strategy decreases first and then increases. The probability of Internet media choosing the publishing strategy decreases due to the increase in $W^{M}$ at the beginning. Internet media would choose no-publishing strategy at this time. Then, the probability of Internet users choosing concerning strategy increases to 1 quickly. The strategy of Internet media changes into publishing strategy quickly due to the increase in the probability of Internet users choosing the concerning strategy. When $W^{M}$ is more than 250 , the strategy dynamic evolution is shown in Figure 5(c). At this time, the stable strategy changes. The stable strategy of the system is $(0,1,1)$. The stable strategy of Internet media is no-publishing information. It is because the value of $W^{M}$ is so large that Internet media would not choose the publishing strategy even if Internet users would choose the concerning strategy.

From the above analysis, we know that $W^{M}$ has influence on the stable strategy. When $W^{M}$ is smaller, the strategy of Internet media mainly depends on the strategy of Internet users; when $W^{M}$ is larger than a threshold value, the strategy of Internet media will change. Government may set the value to control the development of Internet public opinion.

3.3. The Influence Analysis of $R_{2}^{H}$ on Strategy. The gain $R_{2}^{H}$ has an important impact on the stability strategy of Internet user. To analyze the effect of the value on the stability strategy, the value of $R_{2}^{H}$ is changed without changing other 


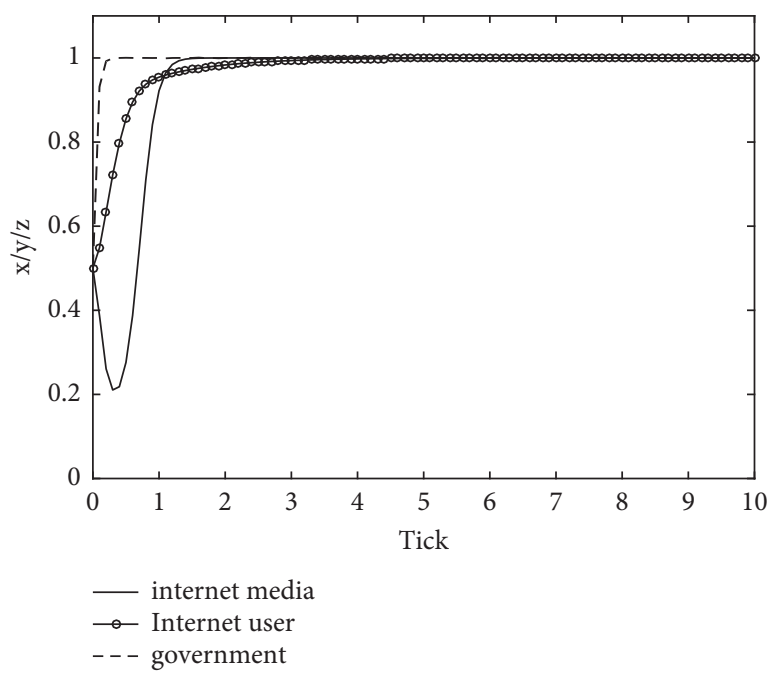

(a)

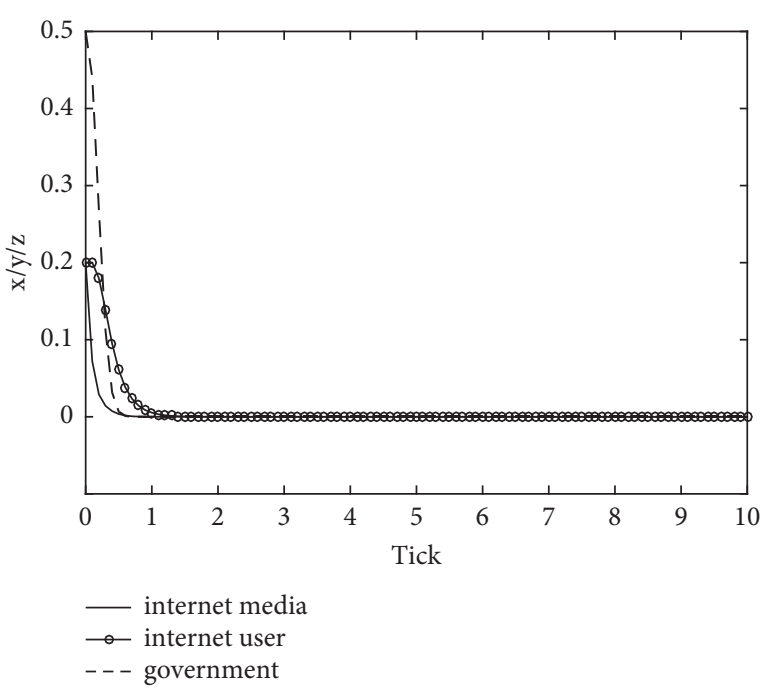

(b)

Figure 4: Strategy dynamic evolution of different initial probabilities: (a) $x=0.5, y=0.5$, and $z=0.5$; (b) $x=0.2, y=0.2$, and $z=0.5$.

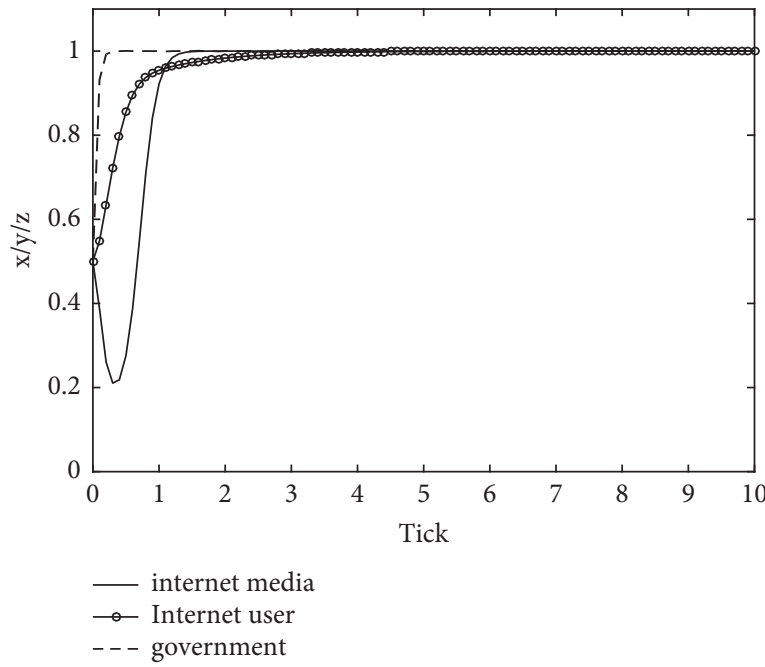

(a)

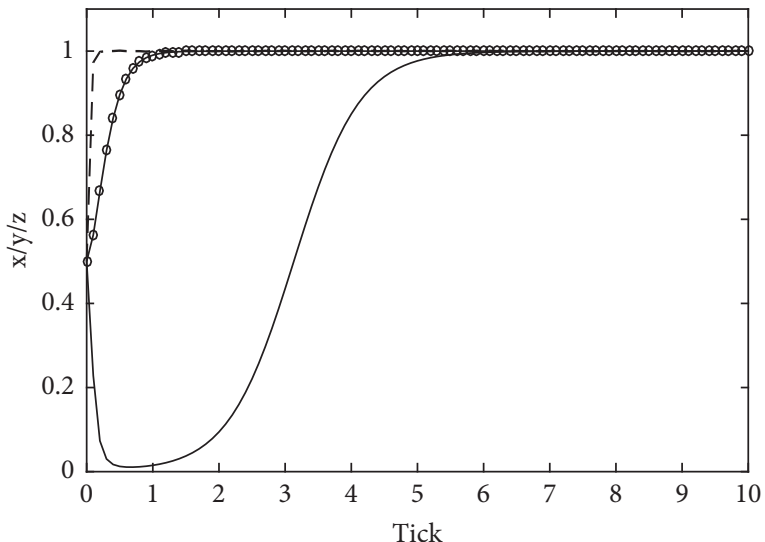

- internet media

$\rightarrow$ internet user - - - government

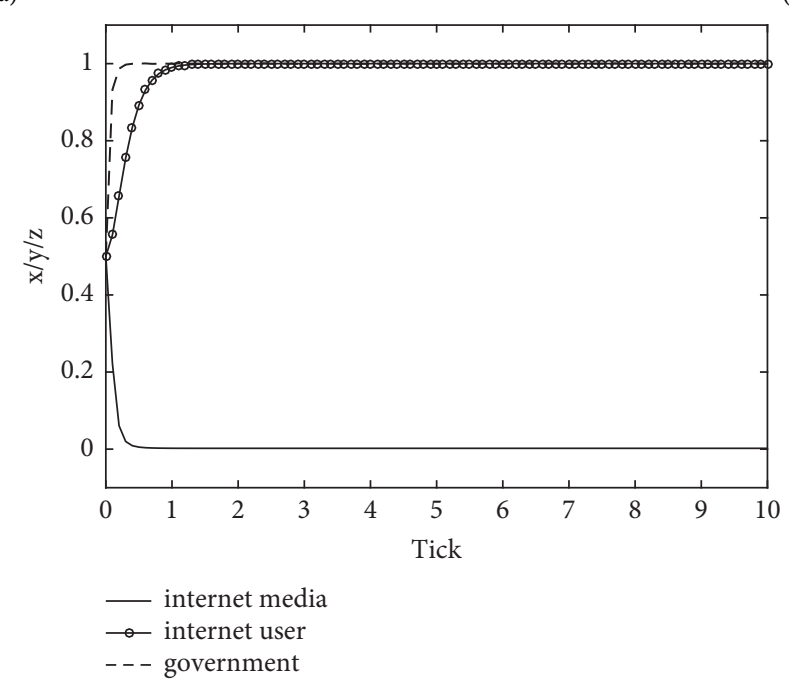

(b)

(c)

FIGURE 5: Strategy dynamic evolution with different $W^{M}$ : (a) $W^{M}<250$; (b) $W^{M}=250$; (c) $W^{M}>250$. 


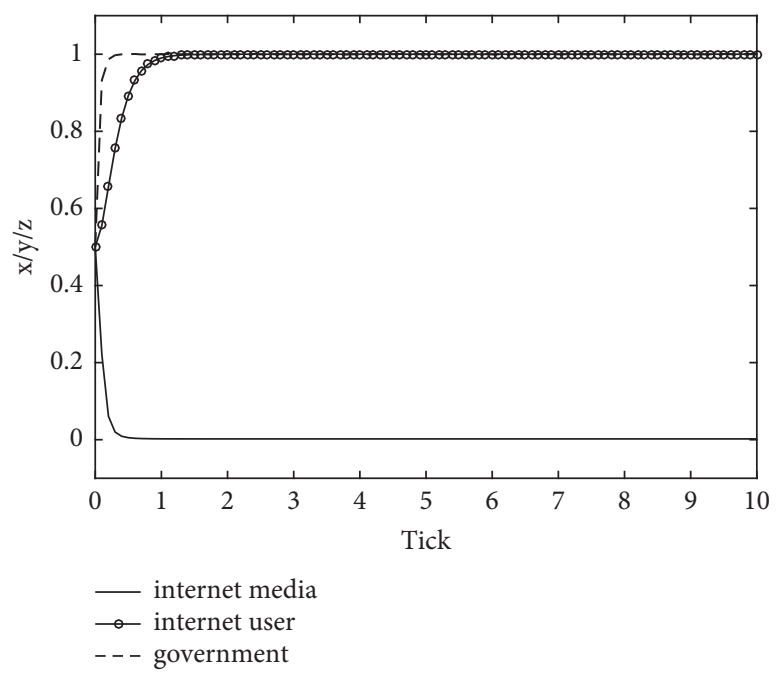

(a)

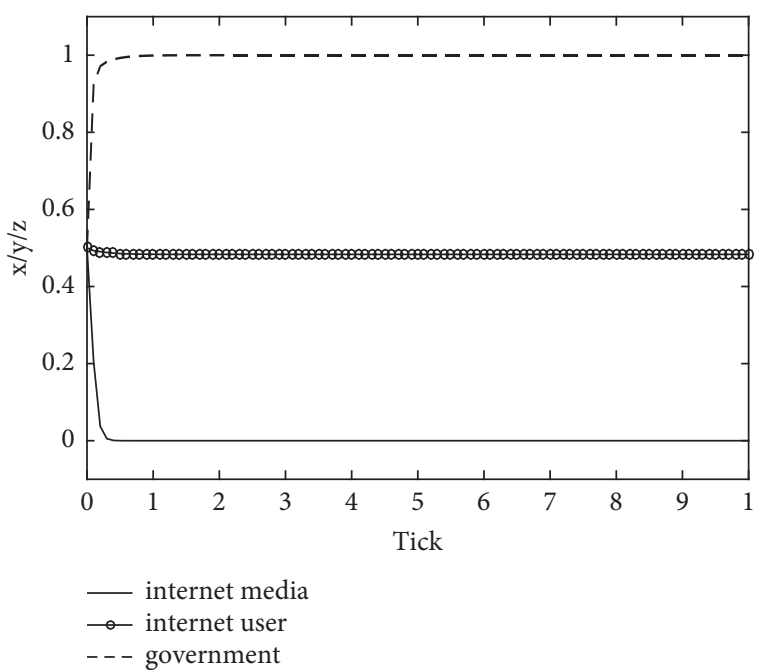

(b)

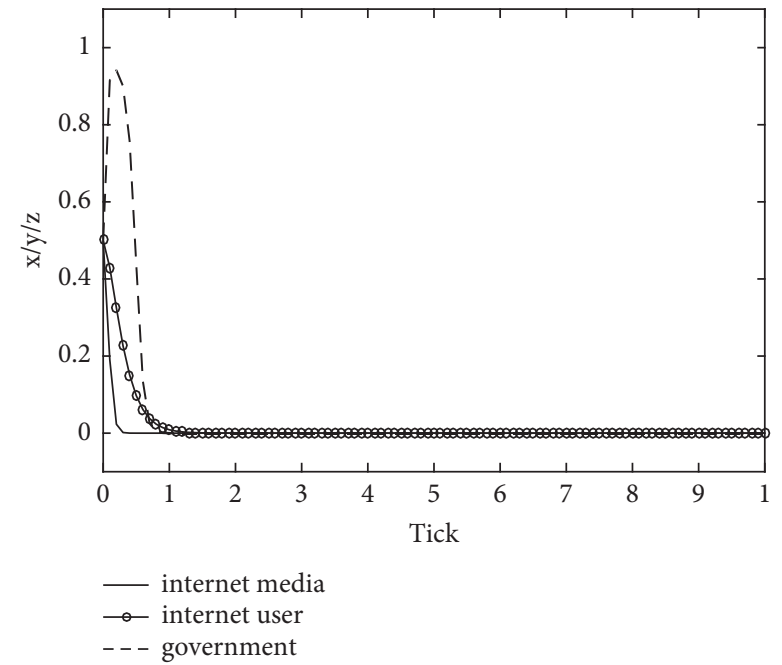

(c)

Figure 6: Strategy dynamic evolution with different $R_{2}^{H}$ : (a) $R_{2}^{H}>50$; (b) $R_{2}^{H}=50$; (c) $R_{2}^{H}<50$.

conditions. When $R_{2}^{H}$ is more than 50 , the strategy dynamic evolution is shown in Figure 6(a). The stable strategy of the system is $(0,1,1)$. When $R_{2}^{H}$ is decreased to 50 , the strategy dynamic evolution is shown in Figure 6(b). The probability of Internet users choosing concerning strategy changes from 1 to 0.5 . When $R_{2}^{H}$ is lower than 50 , the strategy dynamic evolution is shown in Figure 6(c). The stable strategy of the system is $(0,0,0)$. The stable strategy of Internet users is noconcerning information. The strategy of government also changes. The probability of the government choosing the controlling strategy increases first and then decreases. At the beginning, there is a proportion of Internet users choosing concerning strategy and Internet medias publishing information although the proportion is low. Government will still take measures to control it. With the decreasing enthusiasm of Internet media and Internet user for information, it is not necessary to control for the government, so the probability of the government choosing the controlling strategy decreases sharply to 0 . Therefore, the stable strategy of government is no-controlling strategy eventually. In this case, Internet public opinion subsides easily.

From the above analysis, we know that $R_{2}^{H}$ has influence on the stable strategy. When $R_{2}^{H}$ is larger, Internet user easily tends to the concerning strategy; when $R_{2}^{H}$ is equal to a threshold, the probability of Internet users choosing the concerning strategy is 0.5 ; when $R_{2}^{H}$ is smaller than the threshold, Internet user easily tends to no-concerning strategy.

3.4. The Influence Analysis of $C^{G}$ on Strategy. The cost $C^{G}$ has an important impact on the stability strategy. To analyze the effect of the value on the stability strategy, the value of $C^{G}$ is changed without changing other conditions. When $C^{G}$ is lower than 500, the strategy dynamic evolution is shown in Figure $7(\mathrm{a})$. The stable strategy of the system is $(1,1,1)$. When $C^{G}$ is more than 500 , the strategy dynamic evolution is shown in Figure 7(b). The stable strategy of the system is (1, 


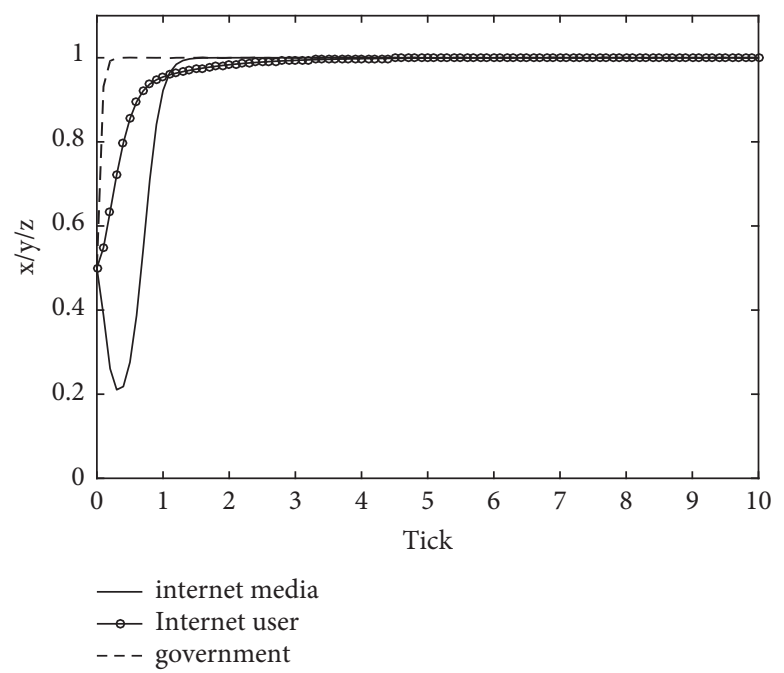

(a)

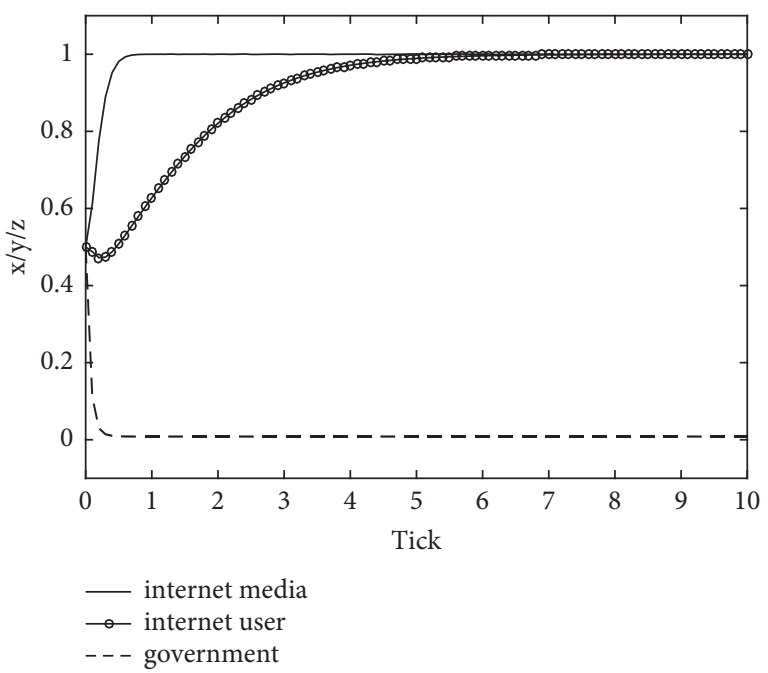

(b)

Figure 7: Strategy dynamic evolution with different $C^{G}$ : (a) $C^{G}<500$; (b) $C^{G}>500$.

$1,0)$. Strategy of government changes into no-controlling strategy due to the increase in the controlling cost. The probability of Internet user choosing concerning strategy shows small decrease first then increase. At the beginning, the probability of the government choosing the controlling strategy is not zero. Internet user benefits from social stability for government controlling strategy. Therefore, some Internet users support the government, and the probability of Internet user choosing concerning information strategy decreases. However, when the probability of the government choosing the controlling strategy reaches to 0 , Internet user is no longer benefiting from government. Therefore, the probability of Internet user choosing concerning information strategy increases to 1 . At this time, Internet public opinion will bring a negative impact on society, with this tendency uncontrolled.

\section{Conclusions}

This paper studies the problem of Internet public opinion evolution from the perspective of evolutionary game theory. A model of Internet public opinion evolution is established considering Internet media, Internet users, and government. The evolution strategy and stability are analyzed. The influences of the strategy are studied by simulation. The corresponding conclusions are obtained as follows:

(1) There are multiple possible stable strategies in the evolution system of Internet public opinion. The stable strategy of the system depends on the initial probability of the strategy and system parameters, such as cost, gain, and loss. The initial probability of strategy in different areas leads to the different stable strategies. The model can help to analyze and forest the development of Internet public opinion based on system parameters and the initial probability of the strategy in a specific environment.
(2) The stable strategy is affected by the punishment parameter $W^{M}$ of Internet media. Government may set the value to control the development of Internet public opinion. When the value is smaller, Internet media will choose the publishing strategy. When the value is more than a threshold, the strategy of Internet media will change into no-publishing strategy. The threshold can be obtained by the method in this paper.

(3) The stable strategy is affected by the gain parameter $R_{2}^{H}$ of Internet user. When the gain Internet users feel from government decreases, Internet users would change the strategy to maintain social stability and find security sense. The threshold of the parameter can be obtained by the method in this paper.

(4) The cost of government $C^{G}$ has an influence on the stable strategy. When the cost is more than a threshold, the government would not choose the controlling strategy. It can result in the uncontrolled development of Internet public opinion. The threshold of cost can be obtained by the evolution model.

The above conclusions reflect the relative characteristics and regulations of Internet public opinion evolution, which can give us some guide significance for the management of Internet public opinion. The works provide the foundation for the research studies on public opinion monitoring and controlling in future. Government is the core of the Internet public opinion evolution system. Government takes the initiative of Internet public opinion evolution. It can control the evolution of Internet public opinion through adjusting the punishment for Internet media, administrative cost, and so on.

From the perspective of the tripartite game, the model considers the evolution of Internet public opinion as the strategy evolution result of the game participants. The 
evolutionary game method is used to analyze the model, revealing the characteristics of bounded rationality. It makes the analysis closer to human characteristics in reality. The model has a certain universal applicability, which can simulate and forecast the evolution result of Internet public opinion in a specific problem. In the future research work, some variables would be considered in the model, such as the impact of opinion leader and media agenda setting.

\section{Data Availability}

The data used to support the findings of this study are included within the article.

\section{Conflicts of Interest}

The authors declare that there are no conflicts of interest regarding the publication of this paper.

\section{Acknowledgments}

This work was supported in part by the National Natural Science Foundation of China under Grants 61963023 and 61841303 and in part by the Tianyou Innovation Team of Lanzhou Jiaotong University under Grant TY202003. The authors express their thanks to all who participated in this research for their cooperation.

\section{References}

[1] Z. Lin, "Online public opinion governance of public emergencies: orientation and research directions," Journal of Intelligence, vol. 38, no. 2, pp. 119-124, 2019.

[2] X. L. Wang and J. Gu, "Research on the diffusion path and vertical spillover effect in supply chains of online public opinion after firm crisis," Journal of Management Science, vol. 32, no. 1, pp. 42-55, 2019.

[3] Y. Mei, Y. Tu, K. F. X, Y. Xie, and W. Shen, "Internet public opinion risk grading under emergency event based on AHPsort II-DEMATEL," Sustainability, vol. 11, no. 16, p. $4440,2019$.

[4] D. Stockmann and T. Luo, "Which social media facilitate online public opinion in China," Problems of Post-Communism, vol. 64, no. 3-4, pp. 189-202, 2017.

[5] K. Silje, H. Bonfadelli, and M. Kovic, "Risk perception of nuclear energy after Fukushima: stability and change in public opinion in Switzerland," International Journal of Public Opinion Research, vol. 30, no. 1, pp. 24-50, 2018.

[6] J. Tian and M. Y. Zhang, "Prism refraction: the generation logic and content hierarchy of network public opinion-analysis of "taxi strike incident" based on grounded theory," Information Science, vol. 37, no. 8, pp. 38-43, 2019.

[7] S. Y. Li and W. L. Li, "Evolution mechanism of public crisis network public opinion: path and motivation-taking animal epidemic crisis as an example," Chinese Public Administration, no. 2, pp. 116-121, 2019.

[8] J. Y. M. Nip and K.-w. Fu, "Challenging official propaganda? Public opinion leaders on Sina Weibo," The China Quarterly, vol. 225, pp. 122-144, 2016.

[9] Y. Z. Hao, Q. H. Zheng, Y. P. Chen, and C. X. Yan, "Recognition of abnormal behavior based on data of public opinion on the web," Journal of Computer Research and Development, vol. 53, no. 3, pp. 611-620, 2016.

[10] E. Haihong, Y. X. Hu, H. P. Peng, W. Zhao, S. Xiao, and P. Niu, "Theme and sentiment analysis model of public opinion dissemination based on generative adversarial network," Chaos, Solitons \& Fractals, vol. 121, pp. 160-167, 2019.

[11] Y. Lian, X. Dong, and Y. Liu, "Topological evolution of the internet public opinion," Physica A: Statistical Mechanics and Its Applications, vol. 486, pp. 567-578, 2017.

[12] G. H. Wang, "Research on network public opinion supervision forecast based on big data analysis," Computer and Modernization, vol. 12, pp. 62-66, 2016.

[13] B. Ma and L. F. Yin, "A parallel naive bayesian network public opinion fast classification algorithm based on hadoop platform," Data Analysis and Knowledge Discovery, vol. 31, no. 2, pp. 78-84, 2015.

[14] R. Guo, H. Li, and F. J. Chen, "Research on network public opinion evolution prediction based on extension clustering," Information Studies: Theory \& Application, vol. 40, no. 1, pp. 83-87, 2017.

[15] G. B. Sandra and P. Georgios, "Signals of public opinion in online communication: a comparison of methods and data sources," The Annals of the American Academy of Political and Social Science, vol. 659, no. 1, pp. 95-107, 2015.

[16] J. K. Wang, H. Yu, and X. H. Wang, "Dissemination and control model of public opinion in online social networks based on users' relative weight," System Engineering-Theory \& Practice, vol. 39, no. 6, pp. 1565-1579, 2019.

[17] C. Ling, J. W. Feng, and P. Wu, "A study on crisis response of campus network public opinion based on SOAR model," Information Science, vol. 37, no. 9, pp. 145-152, 2019.

[18] J. Song, P. Wu, and L. R. Gan, "Modeling and simulating for the public opinion evolution of cellular automata based on netizens' silence factors," Information Studies: Theory \& Application, vol. 38, no. 8, pp. 124-129, 2015.

[19] A. G. Javier, "Network models of minority opinion spreading: using agent-based modeling to study possible scenarios of social contagion," Social Science Computer Review, vol. 34, no. 5, pp. 567-581, 2015.

[20] D. Zhao, X. W. Wang, M. M. Xiang, and M. Q. Yang, "Model of micro-blog public opinion dissemination trend under the new media environment: an information ecology perspective," Journal of Intelligence, vol. 35, no. 10, pp. 173-180, 2016.

[21] H. Z. Zhou and J. Zhang, "Diffusion of self-media network public opinion based on forwarding effect," Control and Decision, vol. 34, no. 3, pp. 572-580, 2019.

[22] Y. Zhao, G. Kou, Y. Peng, and Y. Chen, "Understanding influence power of opinion leaders in e-commerce networks: an opinion dynamics theory perspective," Information Sciences, vol. 426, pp. 131-147, 2018.

[23] A. Ishii and Y. Kawahata, "Opinion dynamics theory for analysis of consensus formation and division of opinion on the internet," 2018, https://arxiv.org/abs/1812.11845.

[24] X. Dong, Y. Lian, D. Li, and Y. Liu, "The application of cobbdouglas function in forecasting the duration of internet public opinions caused by the failure of public policies," Journal of Systems Science and Systems Engineering, vol. 27, no. 5, pp. 632-655, 2018.

[25] Q. Zhang, "A collaborative decision approach for internet public opinion emergency with intuitionistic fuzzy value," International Journal of Management and Fuzzy Systems, vol. 5, no. 1, pp. 1-8, 2019.

[26] V. Sathya, A. Venkataramanan, A. Tiwari, and D. D. Ps, "Ascertaining public opinion through sentiment analysis," in 
Proceedings of the international conference computing methodologies and communication, Erode, India, 2019.

[27] X. J. Hu and Z. J. Wei, "Internet public opinion analysis based on apriori association rule mining," in Proceedings of the IEEE advanced information management communicates electronic and automation control conference, Chongqing, China, 2019.

[28] L. Zhang and N. Zhang, "Effectiveness of trust repair strategies in the crisis of corporate internet public opinion," American Journal of Management Science and Engineering, vol. 5, no. 1, pp. 10-18, 2020.

[29] W. H. Sandholm, "Evolutionary game theory," Complex Social and Behavioral Systems: Game Theory and Agent-Based Models, Springer, Berlin, Germany, pp. 573-608, 2020.

[30] S. Babu and U. Mohan, "An integrated approach to evaluating sustainability in supply chains using evolutionary game theory," Computers \& Operations Research, vol. 89, pp. 269283, 2018.

[31] P. Ji, X. Ma, and G. Li, "Developing green purchasing relationships for the manufacturing industry: an evolutionary game theory perspective," International Journal of Production Economics, vol. 166, pp. 155-162, 2015.

[32] X. Li, R. Huang, J. Dai, J. Li, and Q. Shen, "'Research on the evolutionary game of construction and demolition waste (CDW) recycling units' green behavior, considering remanufacturing capability," International Journal of Environmental Research and Public Health, vol. 18, no. 17, p. 9268, 2021.

[33] H. Long, H. Liu, X. Li, and L. Chen, "An evolutionary game theory study for construction and demolition waste recycling considering green development performance under the Chinese government's reward-penalty mechanism," International Journal of Environmental Research and Public Health, vol. 17, no. 17, p. 6303, 2020. 